\title{
ARCHITECTURES AND SYNCHRONIZATION TECHNIQUES FOR COHERENT DISTRIBUTED REMOTE SENSING SYSTEMS
}

\author{
${ }^{1}$ J. C. Merlano-Duncan, ${ }^{1}$ J. Querol, ${ }^{2}$ A. Camps, ${ }^{1}$ S. Chatzinotas, ${ }^{1}$ B. Ottersten \\ ${ }^{1}$ Interdisciplinary Centre for Security Reliability and Trust (SnT), University of Luxembourg \\ ${ }^{2}$ Unidad María de Maeztu CommSensLab - Dept. of Signal Theory and Communications, Universitat \\ Politécnica de Catalunya-BarcelonaTech and IEEC/CTE-UPC \\ Email: \{juan.duncan, jorge.querol, symeon.chatzinotas, bjorn.ottersten\} @uni.lu; \\ camps@tsc.upc.edu
}

\begin{abstract}
Phase, frequency and time synchronization is a crucial requirement for many applications as such as multi-static remote sensing and distributed beamforming for communications. The literature on the field is very wide, and in some cases, the requirements of the proposed synchronization solution may surpass the ones set by the application itself. Moreover, the synchronization solution becomes even more challenging when the nodes are flying or hovering on aerial or space platforms. In this work, we compare and classify the synchronization technologies available in the literature according to a common proposed framework, and we discuss the considerations of an implementation for distributed remote sensing applications. The general framework considered is based on a distributed collection of autonomous nodes that try to synchronize their clocks with a common reference. Moreover, they can be classified in non-overlapping, adjacent and overlapping frequency band scenarios.
\end{abstract}

Index Terms - Synchronization, multi-static remote sensing systems, distributed beamforming.

\section{INTRODUCTION}

Synchronization in terms of absolute phase, frequency and time for clock or local oscillator signals is essential for multi-static remote sensing systems and it is the main requirement for distributed and collaborative beamforming on the communications field. Achieving precise synchronization is a very challenging task when the signals are generated locally at each of the distributed nodes. The synchronization becomes even more difficult when the geometric distance between the distributed nodes is large in terms of the signal wavelengths and, in particular, when this electrical distance is time-varying due to changes in the conditions of the transmission media. This is the case when the nodes are flying or hovering on aerial or space platforms.
Several works on distributed beamforming have been published in the last decade, and different analytical techniques have been refined, however, there are not many practical implementations even at a research stage. The main limiting factor to make practical implementation feasible is the synchronization under realistic scenarios. In those cases, the level of effort, power consumption, and complexity required for the synchronization purposes can surpass the ones required by the application itself. There have been some proposals for multi-base-station distributed beamforming in the area of mobile communications, however, the aforementioned technical challenges were an impediment for practical implementations, at the same time when other MIMO technologies flourished.

Some of the proposed applications relayed on an implicit synchronization that is made through network backhaul links, which is not always present in practice.

Distributed beamforming has also been receiving increased interest in the last decade in the field of Wireless Sensor Networks due to rapid development in sensor technologies and embedded systems using low power equipment. In these cases of extremely low power applications, the distributed and collaborative beamforming looks more appealing than in other types of wireless networks, in principle by the natural topology of the sensor networks and also by its power constraints [1].

Synchronization of distributed radio systems is a recurring design requirement several fields, with applications in telecommunications and, for example, in very large baseline phase arrays. In the last decades, several synchronization techniques for distributed radio systems have been proposed. In 1968, Thompson et al. [2] discussed, compared and classified the available techniques for reducing propagation induced phase fluctuations in frequency distribution systems and defined the principle of round trip stabilization systems. This work was developed under the context of very long baseline arrays for radio astronomy applications. Those applications can be seen as distributed radio systems, however, the distributed nodes have a fixed position and the synchronization and signal distribution is 
done usually by means of an auxiliary wired or wireless transmission media. The phase synchronization schemes described in [2] can be generalized as distributed Phase Locked Loops (PLL). In those distributed PLLs, a direct feedback action is applied over a two-way transmission media as part of a whole phase loop under the assumption of channel reciprocity. Even in this case, where the radio units have a fixed position and are interconnected with coaxial cables, the design is very challenging due to the constant variations of the electrical length of the transmission media used for the synchronization, which come as a function of the temperature in the cables and other physical parameters that cannot be easily characterized. The synchronization becomes even more challenging when one or more nodes are constantly moving and a wireless media is used for the synchronization link. The main problems found in this case, on top of the mechanical movement of the nodes, are fading, multi-path and non-reciprocity of the channel, which makes a practical implementation of multi-static systems almost impossible in cluttered or indoor radio environments. Another challenge in distributed remote sensing systems is the trade-off between the radio resources, such as power and spectrum, used by the synchronization and coordination mechanism in comparison to the resources used by the sensing process itself.

Under these constraints, we foresee a set of scenarios for which distributed microwavehremote sensing can flourish in future practical applications. The main factor that will benefit the implementation of distributed sensing will be the availability of a cost-efficient inter-node communication channel suitable for synchronization and coordination. One good example of these scenarios are the swarms or formation flying topologies implemented using spaceborne or airborne platforms.

In this paper, we study and compare the available technologies used for synchronization and coordination of nodes in distributed remote sensing applications. Additionally, we propose a general system model and identify preliminary guidelines and critical elements for the implementation of the synchronization mechanisms.

\section{SYSTEM MODEL}

The general system is a distributed collection or array of independent and autonomous radio nodes which, cooperate to perform a coherent remote sensing operation. From a general perspective, the geometry of a distributed array can have the following configurations:

- The nodes in the array have a fixed position. One example of this are the VLA used in Radioastronomy.

- The nodes of the array are moving or flying elements, while the whole array tries to stay at the same position. One example of this can be an array of stratospheric balloons or HAPS.

- The nodes are moving or flying and the whole is following a trajectory or orbit.

The target remote sensing applications can be passive or active. For the case of passive applications, the array performs distributed beamforming on reception, and for the case of the active application, the array performs distributed beamforming on reception and on transmission. For all the cases, active or passive the radio units have reception capabilities and transmission capabilities in order to synchronize the array, where the whole system performs concurrently multi-beam beamforming. Here we do not assume any particular geometrical distribution for the array of $N$ nodes, where each of them has the state variable

$$
\overrightarrow{x_{n}}=\left(\overrightarrow{p_{n}}, \overrightarrow{v_{n}}\right)
$$

where $\overrightarrow{p_{n}}$ and $\overrightarrow{v_{n}}$ are the variables of position and speed of the node $n$ as a function of time. Each of the nodes has the possibility to use a radio channel to communicate with each other in the array. The complex transfer function of these inter-node channels, which can be simplified to a complex coefficient, are given by $\boldsymbol{h}_{\boldsymbol{n} \boldsymbol{m}}$ with $n$ and $m$ as the node subscripts. All these elements are collected in the matrix $\boldsymbol{H}$ Fig. 1. shows a general scheme of the distributed remote sensing system.

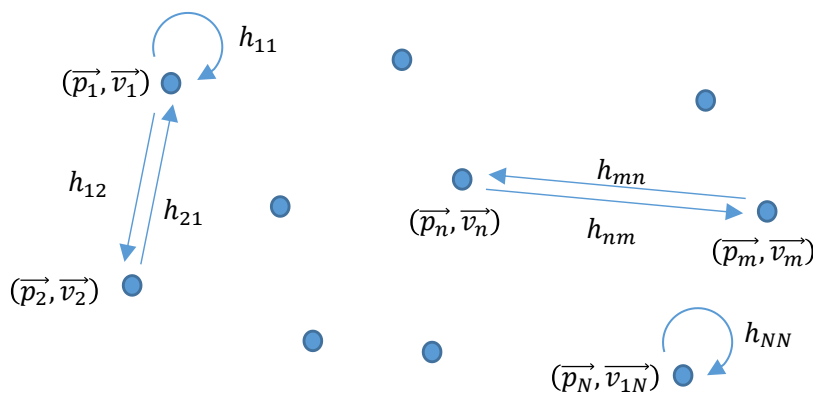

Fig. 1. General scheme for is a distributed array of independent and autonomous nodes performing the required synchronization for a remote sensing operation.

Here we do not assume that high-quality channels are always available among all the nodes. The quality of the internode channels will vary as a function of the position and attitude of the nodes, given that we do not assume that the node radiation patterns are omnidirectional. These channel transfer functions are also affected by the response of all the radio frequency chains inside the nodes.

Each of the nodes generates its own initial time and frequency reference signal $\boldsymbol{c}_{\boldsymbol{n}}(\boldsymbol{t})$. The quality of these initial reference signals is not expected to be of the same quality along with the different array elements. The objective of the 
synchronization system is to lock each of those initial reference signals to the one with the best accuracy, known as a master reference.

In order to achieve this requirement, the set of nodes radiate the complex signals $\boldsymbol{x}_{\boldsymbol{n} \boldsymbol{m}}$, for each $\boldsymbol{n}, \boldsymbol{m}$ combination. Finally, the signals received at each of the nodes are affected by a zero mean independent circularly symmetric complex Gaussian noise, $\boldsymbol{w}_{\boldsymbol{n}}$.

\section{SYSTEM CLASSIFICATION}

The general system can be classified on how the radio resources are split among the internode communication and the remote sensing beamforming operation as follows:

- Non-overlapping band scenarios: Here the frequencies used for internode synchronization are in a different frequency band to the band used for the remote sensing operation. In these scenarios, the reference signals inside each of the nodes can track the master reference, but a divergence of the absolute phases can appear since the phase variations at the remote sensing frequencies are not completely tracked with the internode links. Some examples of these kind scenarios are described in [3],[4], $[5],[6]$.

- Adjacent frequency scenarios: Here the sensing operation and the internode connection use the same band, but with different central frequencies. In this case, the phase absolute phase variation at the application frequencies is tracked more accurately in comparison to the previous case.

- Overlapping scenarios: In this case, the frequencies used for internode synchronization and the sensing application are completely overlapped in frequency. This scenario has the advantage to track all the phase variations occurring in the application frequencies. However, it comes at the price of requiring an additional mechanism to distinguish between the two kinds of signals, either using time multiplexing or using multi signal / multi-user processing approaches. Previous works demonstrated experimentally that in-band full duplex and self-signal cancellation techniques work satisfactorily under high SNR regimes and under an appropriate phase tracking [7][8].

\section{SOME LITERATURE EXAMPLES OF DISTRIBUTED REMOTE SENSING}

- Tandem-X: This mission consists of two SAR satellites following an orbit in close formation, with a variable distance between them of few hundreds of meters [5],[6]. The system produces high-resolution SAR images in Xband in both, monostatic and bistatic configurations. For the bistatic configurations, one of the two satellites operates as transmitter and the other one as receiver. For the bistatic configuration, the system performs a round trip synchronization scheme in order to obtain the coherency required for this configuration. The synchronization scheme is described in the patent [9].

- Low-frequency distributed radio telescope in space (OLFAR) [10],[11]: The Orbiting Low-Frequency Antennas for Radio Astronomy (OLFAR) develops a space-based low-frequency radio telescope that explore the universe's so-called dark ages. The telescope is composed of a swarm of at least fifty satellites.

- LISA: stands for Laser Interferometer Space Antenna [12]. The objective of the mission is to detect and observe the gravitational waves coming from galactic and extra galactic sources, whereas laser interferometry is used to measure the distance with a required accuracy of $20 \mathrm{pm}$.

- GRACE: The Gravity Recovery and Climate Experiment was a mission to track changes in the Earth's gravity field [13]. GRACE consisted of two identical satellites in near-circular orbits at $\sim 500 \mathrm{~km}$ linked by an accurate inter-satellite link [14].

\section{SIMULATION RESULTS}

We perform some simulations of the proposed system model considering six nodes. We make use of orthogonal sequences with unitary power, we assume the noise at the receivers to be Gaussian with zero mean and unit variance, and we repeat for random/arbitrary symmetric matrices $\boldsymbol{H}$. We that the first node is the phase reference. The slave nodes can get synchronization from the reference using the direct path, which is the first column and first row of the matrix $\boldsymbol{H}$. This synchronization relies on the use of different orthogonal sequences (usually pseudo-random codes). First, the reference node broadcasts one of the sequences providing open-loop synchronization. Then, another pair of sequences is transmitted between reference and slave nodes for each closed-loop synchronization. We show, as an example, the performance of the system using the symmetric matrix:

$$
\boldsymbol{H} \mid=\left[\begin{array}{cccccc}
\mathbf{1} & 0.3 & 0.21 & 0.17 & 0.37 & 0.32 \\
0.3 & \mathbf{1} & 0.54 & 1.02 & 0.32 & 0.78 \\
0.21 & 0.54 & \mathbf{1} & 0.32 & 0.45 & 0.43 \\
0.17 & 1.02 & 0.32 & \mathbf{1} & 0.62 & 0.41 \\
0.37 & 0.32 & 0.45 & 0.62 & \mathbf{1} & 0.31 \\
0.32 & 0.78 & 0.43 & 0.41 & 0.31 & \mathbf{1}
\end{array}\right]
$$

The accuracy of the single synchronization links (master to slaves); using a simple integral control with integral parameter of $\kappa=200$ is shown in Fig. 2. 


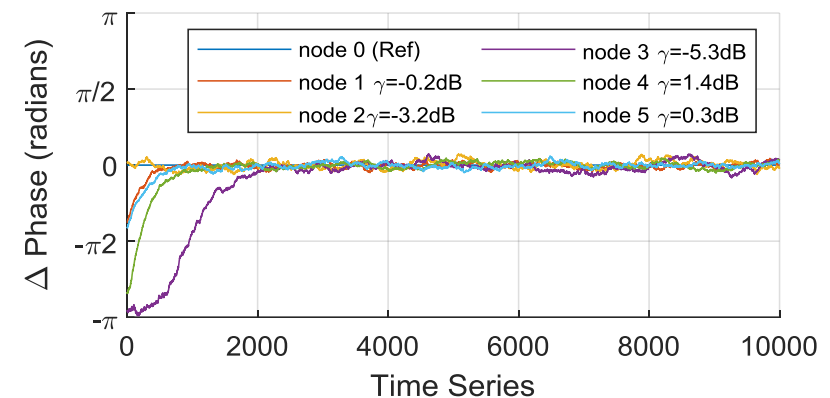

Fig. 2. Time series of the synchronization of point-to-point nodes. $\gamma$ is the received SNR of each individual link. (The transmitted SNR is set to $10 \mathrm{~dB}$ for all the nodes.). The variance of the phase estimator is $\sim 1 /(\kappa \gamma)$.

One-step further, a coordinated approach can be followed taking into account all the possible combinations between nodes and having the phases $\phi$ at each of the nodes as state variables. Assuming that the transmitter pre-compensates the propagation phase, the observed complex value at each of the receivers $n$ used to compensate its phase at a given instant is

$$
\Psi_{n}=\left[\left|h_{0 n}\right| e^{j \phi_{0}}+\sum_{\substack{m \neq n \\ m \neq 0}}^{N}\left|h_{m n}\right| e^{j \phi_{m}}\right] e^{-j \phi_{n}}
$$

The result, also for an integral controller using the parameter $\Psi$ is the one shown in Fig. 3.

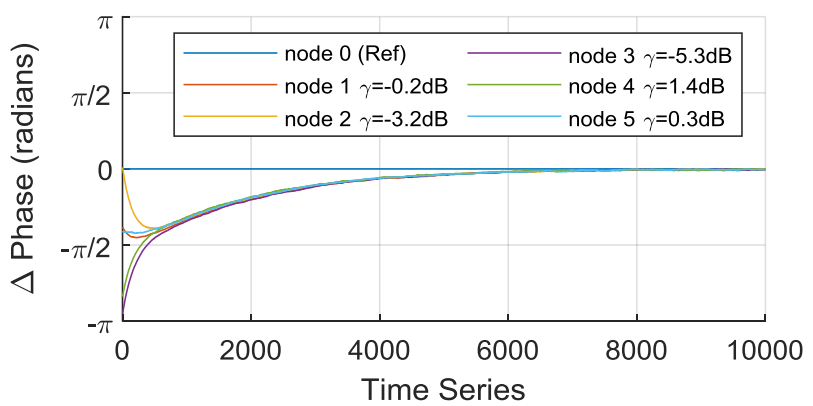

Fig. 3. Time series of the synchronization of multiple coordinated nodes. $\gamma$ is the SNR of each master/slave link.

\section{CONCLUSIONS}

This paper presents a review of different techniques for clock synchronization for remote sensing systems. Moreover, a general model for the synchronization scheme and its preliminary results show how coordinated synchronization schemes perform better than point-to-point systems.

\section{ACKNOWLEDGMENTS}

This work was supported by the Fond National de la Recherche Luxembourg, under the CORE project COHESAT: Cognitive Cohesive Networks of Distributed Units for Active and Passive Space Applications.

\section{REFERENCES}

[1] S. Jayaprakasam, S. K. A. Rahim, and C. Y. Leow, "Distributed and Collaborative Beamforming in Wireless Sensor Networks: Classifications, Trends, and Research Directions," IEEE Commun. Surv. Tutorials, vol. 19, no. 4, pp. 2092-2116, 2017.

[2] J. Thompson M. et al., "Phase stabilization of widely separated oscillators," Antennas Propagation, IEEE Trans., vol. 16, no. 6, pp. 683-688, Nov. 1968.

[3] J. C. Merlano Duncan, "Phase synchronization scheme for very long baseline coherent arrays," TDX (Tesis Dr. en Xarxa), Jul. 2012.

[4] J. C. Merlano-Duncan, J. J. Mallorquí, and P. LópezDekker, "Carrier phase synchronization scheme for very long baseline coherent arrays," Electron. Lett., vol. 48, no. 15 , p. $950,2012$.

[5] H. Fiedler et al., "The TanDEM-X mission: an overview," in 2008 International Conference on Radar, 2008, pp. 60-64.

[6] M. Younis, R. Metzig, and G. Krieger, "Performance Prediction of a Phase Synchronization Link for Bistatic SAR," IEEE Geosci. Remote Sens. Lett., vol. 3, no. 3, pp. 429-433, Jul. 2006.

[7] C. Politis, S. Maleki, J. M. Duncan, J. Krivochiza, S. Chatzinotas, and B. Ottesten, "SDR Implementation of a Testbed for Real-Time Interference Detection With Signal Cancellation," IEEE Access, vol. 6, pp. 2080720821, 2018.

[8] I. R. Pérez et al., "Calibration of correlation radiometers using pseudo-random noise signals," Sensors, vol. 9, no. 8, pp. 6131-6149, 2009.

[9] "Method for drift compensation with radar measurements with the aid of reference radar signals," Oct. 2004.

[10] A. Budianu et al., "OLFAR: Adaptive topology for satellite swarms." International Astronautical Federation (IAF), pp. 1-9, 03-Oct-2011.

[11] S. P. Chepuri, R. T. Rajan, G. Leus, and A.-J. van der Veen, "Joint Clock Synchronization and Ranging: Asymmetrical Time-Stamping and Passive Listening," IEEE Signal Process. Lett., vol. 20, no. 1, pp. 51-54, Jan. 2013.

[12] "LISA - Laser Interferometer Space Antenna - NASA Home Page." [Online]. Available: https://lisa.nasa.gov/. [Accessed: 07-Jan-2019].

[13] B. D. Tapley, S. Bettadpur, M. Watkins, and C. Reigber, "The gravity recovery and climate experiment: Mission overview and early results," Geophys. Res. Lett., vol. 31, no. 9, p. n/a-n/a, May 2004.

[14] J. Kim and B. D. Tapley, "Simulation of dual one-way ranging measurements," in J. Spacecr. Rockets, 2003, vol. 40, no. 3, pp. 419-425. 